\title{
Automotive Grid Malaysia Experience
}

\section{Saliza Hasan}

Advanced Computing Lab (Grid Computing Lab)

MIMOS Berhad, Technology Park Malaysia, 57000 Kuala Lumpur, Malaysia

E-mail: saliza.hasan@mimos.my

\section{Mohammad Fairus Khalid}

Advanced Computing Lab (Grid Computing Lab)

MIMOS Berhad, Technology Park Malaysia, 57000 Kuala Lumpur, Malaysia

E-mail: fairus.khalidemimos.my

\section{Shahrir Abdullah}

Centre for Automotive Research, Faculty of Engineering and Built Environment Universiti Kebangsaan Malaysia, 43600 UKM Bangi, Selangor, Malaysia

E-mail: shahrireukm.my

\begin{abstract}
Computer Aided Engineering (CAE) design tools are not new in the automotive industry. These tools together with good facility in term of compute, storage and network will help to accelerate designers and researchers in their works. Having such environment for academic and research purpose is requiring huge investment. In Malaysia a group of institutions consist of local universities i.e. Universiti Kebangsaan Malaysia (UKM), Universiti Malaya (UM), Universiti Putra Malaysia (UPM), Universiti Teknologi Malaysia (UTM) and Universiti Kuala Lumpur (UniKL) with collaboration of MIMOS Berhad and Perusahaan Otomobile Nasional Sdn Bhd (PROTON), headed by UKM embark in a project called Computationally Optimised Fuel-Efficient Concept Car (COFEC). This project focuses on using the grid computing as the collaborative and integration platform for the concept car research in virtual environment. In this paper we will share our experience and how we overcome some challenges in building such platform.
\end{abstract}


The International Symposium on Grids and Clouds (ISGC) 2012 Academia Sinica, Taipei, Taiwan

February 26-March 2, 2012 


\section{Introduction}

Automotive Grid is a research project funded by Malaysian Government. The project goal is to develop a computational optimized fuel-efficient concept car through the use of Computer Aided Engineering (CAE) tools. The parties involved in this project belong to different institutes and are geographically dispersed all over Malaysia. Different researches are working on different area and later they need to integrate their works. One of the main challenges for this project is to coordinate the research work in term of High Performance (HPC) resources used in this project. For every institute to have their own big compute infrastructure will not be practical. It is best for the researchers to be able to share their resources and take turn to use the infrastructure in doing their research.

For this reason one of the deliverable of this project is to come up with a shareable platform. The project members agreed to have a grid computing environment by utilizing the gLite as the middleware for the grid setup.

In this paper we will share our experience in setting up the grid platform for this project.

Section 2 describes an overview and objectives of the Automotive Grid project. Section 3 explains the proposed infrastructure setup and several CAE applications installed for this project. Section 4 discusses on the challenges and section 5 we will share the outcome of the project. Lastly, we present our summary.

\section{Automotive Grid Project}

\subsection{Background}

Automotive Grid project is a multi-institutional project which gathers seven local institutes of higher education, $R \& D$ organization and industry, namely Universiti Kebangsaan Malaysia (UKM), Universiti Malaya (UM), Universiti Putra Malaysia(UPM), Universiti Teknologi Malaysia(UTM), Universiti Kuala Lumpur (UniKL), MIMOS and Perusahaan Otomobil Nasional Sdn. Bhd. (Proton). The official project name is Computationally Optimised FuelEfficient Concept Car (COFEC) and it is lead by Prof. Ir. Dr. Shahrir Abdullah from UKM.

MIMOS role in this project is to provide infrastructure consultancy and grid computing facility. The sub project for MIMOS is called "Development of Automotive Grid for KnowledgeGRID ${ }^{\text {TM }}$ Malaysia".

This sub project will support seven other sub projects under COFEC. They are, "Adaptive Heat Transfer and Aerodynamic Models for Integrated Engine Cooling and Vehicle Aerodynamic Simulation" lead by UKM, "Time-based / Transient Simulation of Engine Coolant Peak Temperature After Key-off" lead by UM, "Simple Durability Programming, Integrate with LS-Dyna Post-processing" lead by UPM, "Damage and Fracture MechanicsBased Design Methodology" lead by UTM, "Prediction and Solution of Springback in Metal Forming" lead by UniKL, "Robust Design of Automotive Component using Optimization Analysis in Nonlinear Domain" lead by UniKL and "Formulating Optimization Method for the Best Ride and Handling for Passive Suspension Systems" lead by UM. 


\subsection{Objectives}

At the beginning of the project several objectives have been identified by the project team member. As for the sub project handled by MIMOS, the core function is to provide computing power and resources to all automotive researchers. There are 3 objectives set for this sub project:

a. To develop computationally optimized fuel-efficient concept car in virtual environment. ${ }^{1}$

b. To develop CAE modules and optimization tools for component manufacturing and assembly. ${ }^{1}$

c. To achieve a nearly virtual approach in developing new vehicles by utilizing CAD/CAE and Grid \& High Performance Computing technologies. ${ }^{1}$

\section{Collaboration Platform}

High Performance Computing setup is not new to the project collaborators. They are using the environment for their research work. Unfortunately the HPC setup is configuring as a silo. It works as individual cluster of computing element. In this setup, researchers will not be able to make use of each other resources and each of them having their own access control. In meeting the objectives of the project we are proposing a system that can integrate this distributed resources and at the same time provides fair resource allocation for the researchers to use. One clear choice for such system is the grid computing setup.

\subsection{Deployment of Grid Computing Sites Middleware}

MIMOS started involvement in grid computing since early 2006. We established our first grid setup base on Globus middleware and we have our resources connected to Pacific Rim Application and Grid Middleware Assembly (PRAGMA) infrastucture. By the end of 2008 we started to learn about gLite middleware and involved with Enabling Grids for E-SciencE (EGEE). In March 2009, we have our site certified as part of EGEE infrastructure. In the same year we also took part in the EUAsiaGrid project. Throughout the EUAsiaGrid project, a couple of other institutes in Malaysia they are UPM and UM also established connectivity to the EGEE infrastructure. gLite middleware is distributed under open source license and integrated components from the best of current middleware projects, such as Condor and the Globus Toolkit, as well as components developed for the LCG project ${ }^{2}$. Through this experience and understanding we decided to use gLite as a standard middleware for the Automotive Grid platform.

In addition to the MIMOS's gLite setup that connected to EGEE infrastructure, MIMOS also has established a gLite setup with full services that capable to operate by itself. In other words the setup has its own autonomy which is not connected to the EGEE. The COFEC project is a closed project, accessible by the members only and it doesn't require stringent Service Level Agreement like the EGEE infrastructure. For this project MIMOS is running all core services of the gLite middleware and other institutes will connect to MIMOS setup. 
For this setup, other institutes have two options to connect to MIMOS infrastructure. Figure 1 below depicts the two options. In both of this option gLite user authentication and authorization will be handled by MIMOS infrastructure.

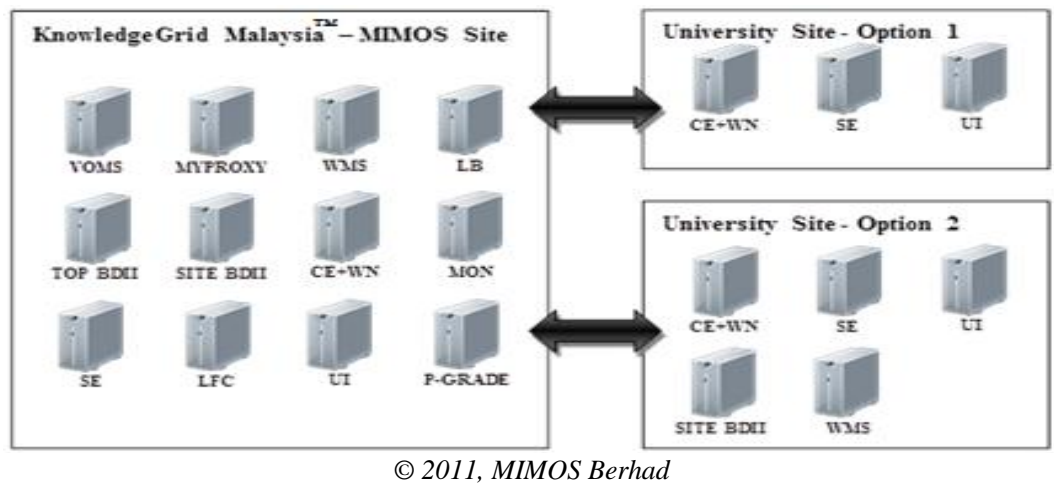

Figure 1: Proposed gLite Site Development

Option 1 is the minimum setup that a site can have. In this setup the institutes are required to provide Compute Element/Worker Nodes (CE/WN), Storage Element (SE) and User Interface (UI). A user can use the UI in their site to submit their job to their local resources and if their want to submit their job into different site they will need to use the Workload Management System (WMS) at MIMOS site. In this setup the site will have a limitation to expend and reduce the number of resources that contribute to the grid. Each time they want to change this they will have to update MIMOS.

Option 2 will enable the user to have more control on their site. With this setup they can simply add more Compute Element(CE) as long as they want. The setup consists of Workload Management System (WMS), Site BDII, Compute Element/Worker Nodes (CE/WN), Storage Element (SE) and User Interface (UI).

\subsection{Applications and Software}

Grid setup by itself is just a list of hardware connected through a middleware. To perform computationally fuel-efficient concept car analysis several applications need to be installed at the KnowledgeGRID ${ }^{\mathrm{TM}}$ Malaysia platform. The applications that have been installed for Automotive Grid are STAR CCM+, ModeFrontier, OpenFOAM, LS DYNA, Hyperworks and Abaqus.

STAR-CCM+ provides the world's most comprehensive engineering simulation inside a single integrated package ${ }^{3}$. Much more than just a CFD solver, STAR-CCM+ is an entire engineering process for solving problems involving flow (of fluids or solids), heat transfer and stress $^{3}$. For COFEC project, STAR CCM+ software is used for full vehicle simulation such as for vehicle underhood thermal analysis, underhood flow field and underhood total pressure analysis. Subprojects under COFEC that used the STAR CCM+ software are Adaptive Heat Transfer and Aerodynamic Models for Integrated Engine Cooling and Vehicle Aerodynamic from UKM and Time-Based/Transient Simulation of Engine Coolant Peak Temperature After Key-Off from UM. 
HyperWorks provides the most comprehensive, open-architecture CAE solution in the industry, including the best-in-class modeling, analysis, visualization and data management solutions for linear, nonlinear, structural optimization, fluid-structure interaction, and multibody dynamics applications ${ }^{4}$. Under COFEC project, Hyperworks software is used for geometric modelling \& meshing by subproject Robust Design of Automotive Component Using Optimization Analysis in Non-Linear Domain lead by UniKL.

OpenFOAM has an extensive range of features to solve anything from complex fluid flows involving chemical reactions, turbulence and heat transfer, to solid dynamics and electromagnetics ${ }^{5}$. COFEC project use OpenFoam software for $\mathrm{C}++$ code development.

ModeFRONTIER refers to the so-called "Pareto Frontier": it is the ideal limit beyond which every further implementation compromises the system; in the other words, it represents the set of best solutions possible ${ }^{6}$. ModeFRONTIER software is used by COFEC project in integration part and development of computer algorithm/formulation for optimization based on ride and handling of full vehicle model (FVM). This software is used by subproject from UM titled Formulating Optimization Method for the Best Ride and Handling for Passive Suspension Systems.

LS-DYNA is developed as a strongly coupled multi-physics solver, rather than loosely coupled field equations ${ }^{7}$. A combined Implicit/Explicit solver - one scalable code for solving highly nonlinear transient problems enabling the solution of coupled multi-physics and multistage problems ${ }^{7}$. In COFEC project, LS DYNA is used for integration with algorithm durability code which is written in Fortran. LS DYNA software is used by subproject from UPM under title Simple Durability Programming Integrate with Ls-dyna Post Processing.

These applications have been installed at different cluster at MIMOS based on CPU's requirement.

\section{Challenges and Issues}

The key success of any project is about how we handle the challenges and issues raised during the project deployment. We organize the problems into two major categories, namely infrastructure and applications.

\subsection{Infrastructure}

Implementation of the infrastructure setup follows the Grid and HPC standard defined by OpenGRID Forum. This setup consists of four main layers comprising of user interface layer (application and serviceware), middleware layer, resources layer and network layer. Among the issues that we face during the implementation of Automotive Grid initiative are resources, CPU utilization, SLA, IP Address, organization policy and security.

\subsubsection{Resources}

Nature of projects is related to the automotive research area, issues in aerodynamics, crash, structural durability, noise and vibration are among the main area to be explored. Therefore, this project aimed to prepare a high computing platform to run the CAE simulation for the selected automotive research area in a virtual environment with less dependent on the real experiments. 
However to setup a computing platform for automotive research community at university required the dedicated grid computing resources in terms of computing power (HPC), CPU, data storage, high bandwidth and memory to be placed. As a solution, minimum requirement of gLite site consist of CE, SE and UI connected with existing VO at MIMOS has been proposed to be setup at university. For the resources monitoring, we use gLite Information Services (IS). gLite IS provide a mechanism to publish and consume information for monitoring purposes. The information and monitoring system can be used directly to publish resources information on the Grid infrastructure. The resource information from gLite IS can be viewed via Parallel Grid Run-time and Application Development Environment (P-GRADE) portal.

\subsubsection{CPU Utilization}

Automotive Grid project needs the high CPU utilization to run the CAE simulation. To allow each user to have equal access in using the grid computing facilities at MIMOS, the numbers of available cores per run per user need to be set accordingly in order to cope with the increasing jobs to be submitted. This measure is necessary so that all users have an equal chance to run their jobs without queuing for too long.

\subsubsection{Service Level Agreement}

Efficient resource management is one of the most important task dealt with high computing power. The power failure or cluster downtime will give significant impact on the user access. Service Level Agreements play a crucial role for the project that comprising of multi institutional in order to provide quality of guaranteed service. Since MIMOS is currently operating a non-commercial grid, the services offered are based on best effort.

\subsubsection{IP Address}

IP address is among the issue that we face during connection setup between KnowlegeGRID ${ }^{\mathrm{TM}}$ Malaysia infrastructure and universities because most of the universities use the dynamic IP address. The dynamic IP addresses are most frequently assigned on LANs and broadband networks by Dynamic Host Configuration Protocol (DHCP) servers because it avoids the administrative burden in assigning the specific static addresses to each device on a network. To allow access to MIMOS Grid Computing servers, COFEC members require to get static IP address to establish the connection.

There are procedures that need to be followed before connectivity from universities to KnowlegeGRID $^{\mathrm{TM}}$ Malaysia infrastructure can be established. To overcome this issue, universities are suggested to use NAT IP Address to limit the number of public IP addresses for both economy and security purposes.

\subsubsection{Organization Policy on the internet/ firewall Port}

Many organizations have a network perimeter firewall that is designed to prevent the entry of malicious traffic in to the organization's network. We still have to comply the university policy and ensure the gLite sites are accessible from MIMOS vice versa. Once the Fully Qualified Domain Name (FQDN) for the CE/WN, SE and UI already registered in the DNS Server at site, we still facing the problem in accessing the gLite site server. There is another list 
of tcp port that needs to be opened at firewall level from site to establish gLite connection from MIMOS to site.

\subsubsection{Security}

We use Grid Security Infrastructure (GSI) to resolve the security issues. GSI is the core of gLite security services. It enables secure authentication and communication over an open network. GSI is based on public key encryption, X.509 certificates, and the Secure Sockets Layer. In order to authenticate oneself to Grid resources, a user needs to have a digital X.509 certificate issued by a Certification Authority (CA) trusted by infrastructure. The authorization of a user on a specific Grid resource is done through Virtual Organisation Membership Service (VOMS), Local Centre Authorization Service (LCAS) and Local Credential Mapping Service (LCMAPS) mechanism. For security enhancement, the Gridsphere framework has been configure to support for the SSL/TLS protocol by using the certificate based authentication to login and access the whole P-GRADE portal. The trusted roots for the user have been added into the Gridsphere keystore in order for the SSL/TLS mutual authentication to function. This security enhancement enables user to authenticate to P-GRADE portal via SSL certificate based mutual authentication.

\subsection{Application}

In this paper, application setup is the challenges that we would like to address during Automotive Grid project execution.

\subsubsection{Software License}

For Automotive Grid project, software will be installed at MIMOS and utilized by users from various universities at different locations. However, software license is dedicated to the specific user and cannot be transferred or utilized by the others. This issue resolved by purchasing the software license under university and to be placed at Grid Computing facilities of MIMOS. This solution has been implemented for Abaqus and Mode Frontier software. Abaqus is purchased by Universiti Teknologi Malaysia and Mode frontier is purchased by Universiti Malaya. Both softwares are installed at MIMOS and can be accessed by all project members.

\subsubsection{Software License Management}

Currently, user facing difficulty to see how many licenses is available when they submit the job using grid computing facilities. By eliminating the needs of the system administrators to manually track licenses or reconcile against purchases, software license management freeing them for higher value activities and helping the organization keeps the operational costs down.

\subsubsection{Portal}

The interface available for CAE job submission is using the Command Line Interface(CLI) currently. Since not all researchers, especially new users who familiar in using command line, P-GRADE portal for user to submit their automotive job will be setup for this 
project. P-GRADE Portal is a web based, service rich environment for the development, execution and monitoring of workflows and workflow based parameter studies on various grid platforms. P-GRADE Portal hides low-level grid access mechanisms by high-level graphical interfaces, making even non grid expert users capable of defining and executing distributed applications on multi-institutional computing infrastructures.

\section{Result}

In this section, we will explain the result and achievement of Automotive Grid project. Total of 77 research papers, 14 patents and $27 \mathrm{PhD} /$ MASTER students will be generated through this project. The deployment of Grid Computing sites middleware at UKM is the first successful setup in Malaysia for Automotive Grid project.

\subsection{Deployment of Grid Computing Sites Middleware Pilot Project}

As a result of a close collaboration between COFEC members and MIMOS, AutomotiveGRID Lightweight Middleware for Grid Computing (gLite) site will be setup between MIMOS and universities. This site will be linked up with KnowledgeGRID ${ }^{\mathrm{TM}}$ Malaysia -MIMOS Virtual Organizations(VO). Figure 2 shows the diagram on the gLite site development to be installed at university's site.

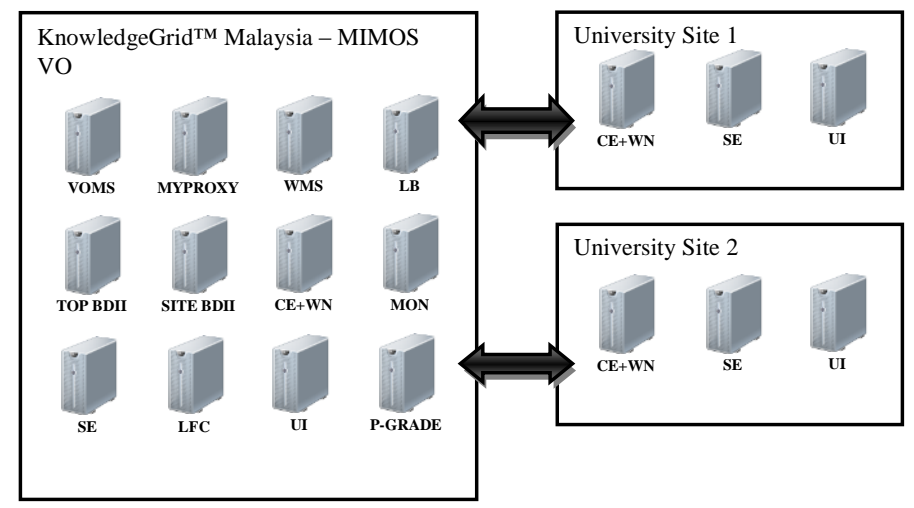

(C) 2011, MIMOS Berhad

Figure 2: KnowledgeGRID ${ }^{\mathrm{TM}}$ Malaysia gLite site development Architecture

Due to limited resources, the minimum gLite components that will be installed at site are Compute Element/Worker Nodes (CE/WN), Storage Element (SE) and User Interface (UI).

At this stage, gLite site development at UKM has been successful setup. For this setup, MIMOS VO infrastructure is being setup at MIMOS data centre while the UKM basic site which hosts the User Interface(UI) is configured at UKM.

Fully Qualified Domain Name (FQDN) for CE, SE, and UI that were set at UKM comprising of grid-ce.ukm.edu.my, grid-se.ukm.edu.my and grid-ui.ukm.edu.my.

Both site UKM and MIMOS need to open their firewall port to establish connection from UKM to MIMOS and vice versa. UKM site must open its SSH port and allow ICMP (ping) from MIMOS server. The other ports that need to be opened are 2170:2172, 20000:25000, $2811,2119,9002,7788,7790$ and 8649. 
The UKM Compute Element (CE) setup can be seen in KnowledgeGRID ${ }^{\mathrm{TM}}$ Malaysia PGRADE portal as shown in Figure 3. Connectivity between both sites is using Malaysia Research and Education Network (MyREN).

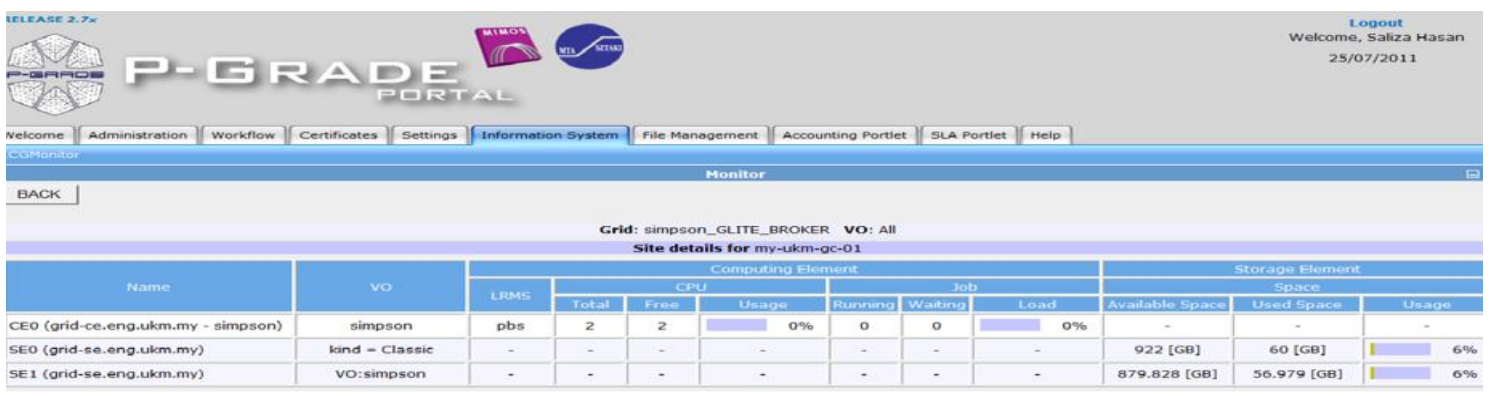

(c) 2011, MIMOS Berhad

Figure 3: KnowledgeGRID ${ }^{\mathrm{TM}}$ Malaysia P-GRADE portal

This deployment is meant for Proof of Concepts (POC). The same component will be configured at Universiti Putra Malaysia (UPM). Future plan is to deploy the site development at all universities involved in this project such as Universiti Malaya (UM), Universiti Teknologi Malaysia(UTM) and Universiti Kuala Lumpur(UniKL).

\subsection{Open Source Software}

Software license required high cost and having restriction in licensing. Because of this issue, open source software like OpenFoam and Scilab have been installed inside gLite infrastructure.

\subsection{User Management}

This project also provide and facilitate users to access the KnowledgeGRID ${ }^{\text {TM }}$ Malaysia infrastructure including user ID and password creation, as well as CAE software and applications to be used by the Automotive Grid users. As a result, total 18 users from UKM, UPM, UM, UTM and UniKL have been created under Automotive Grid project. Automotive Grid users need to have a digital X.509 certificate issued by a Certification Authority (CA) trusted by infrastructure to allow them authenticate to grid computing resources and other services. Grid user will need to contact VOMS server requesting his authorization info and in turn the server sends authorization info to the client, which includes it in a proxy certificate.

\subsection{Resources Utilization by Automotive Grid users}

The infrastructure setup for this project is an ideal platform for automotive research area, especially in running CAE simulation which computing demand is high.

Figure 4 and Figure 5 show the data from SGI ICE and SGI Altix 4700 cluster on the resources utilization by Automotive Grid users. In February 2012, the total is more than 2056 jobs have been submitted with more than 319,615,137 CPUs time (in seconds) have been utilized. 


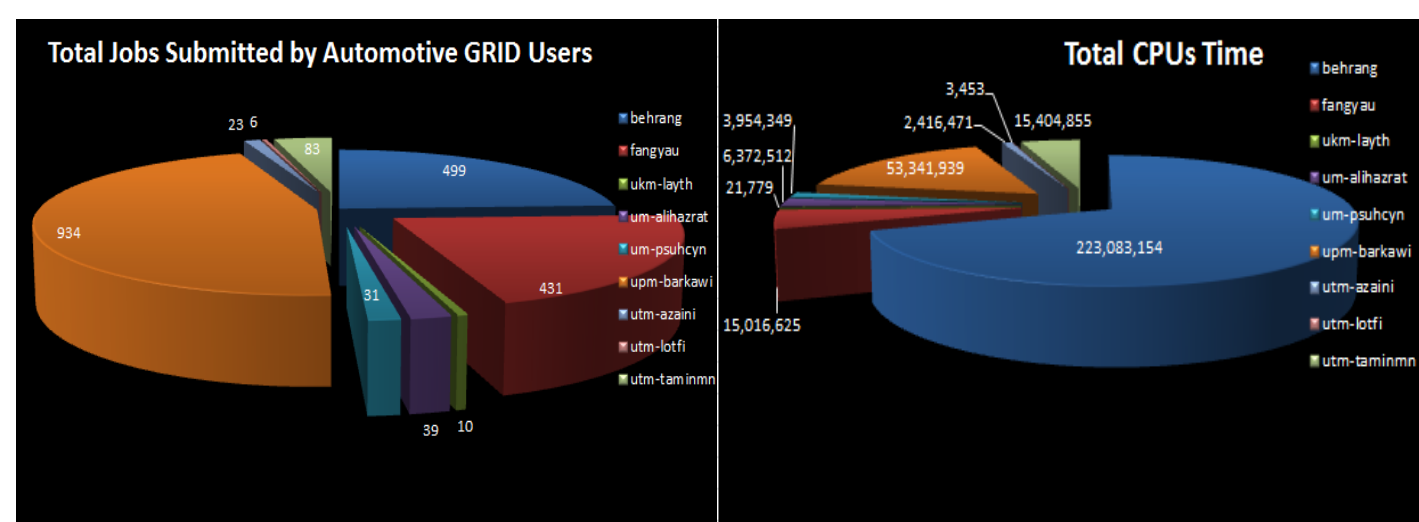

(C) 2011, MIMOS Berhad

Figure 4: Resource Utilization by AutomotiveGRID users - Total Jobs submitted by users
Figure 5: Resources Utilization by

AutomotiveGRID users - Total CPUs Time used by users

\subsection{Sample Output of Simulation from Automotive Grid}

The Figures below are the sample output of simulation from the utilization of the grid computing facilities. By performing CAE analysis virtually, this will assist the automotive researchers to get better result with less dependent on real experiments of initial prototypes. Figures 6 and Figure 7 show the simulation on vehicle underhood thermal analysis using Star$\mathrm{CCM}+$ software. Figure 8 shows the analysis on car fatigue contour using LS DYNA software.

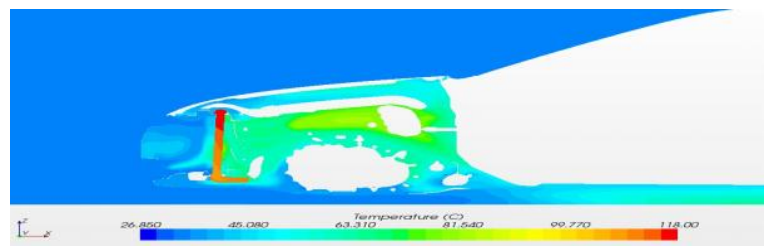

Courtesy from Adaptive Heat Transfer and Aerodynamic Models for Integrated Engine Cooling and Vehicle Aerodynamic Project, UKM

Figure 6: Vehicle Underhood Thermal Analysis using Star-CCM+

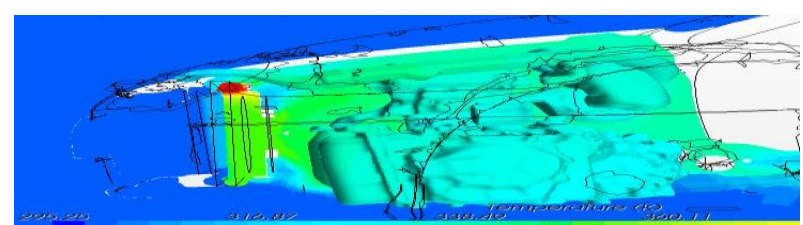

Courtesy from Time-Based/Transient Simulation Of Engine Coolant Peak Temperature After Key-Off Project, $U M$

Figure 7: Under hood Thermal Map Analysis using Star-CCM+

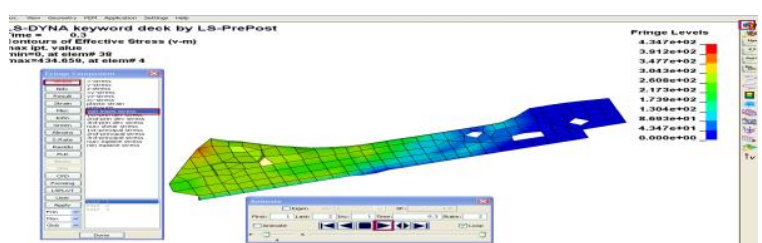

Courtesy from Simple durability programming integrate with LS-DYNA post processing Project, UPM

Figure 8: LS-PrePost - Fatigue Contour Analysis using LS DYNA

\section{Conclusion}


Automotive Grid is aimed to embark in cutting edge research at producing a fuel-efficient concept car by performing CAE analysis on the grid computing facility. In order to obtain an optimised design, the simulation process required excessive computational power, storagehunger and high computational cost. Through the establishment of the first Automotive Grid infrastructure in Malaysia, we hope it will benefit the country especially the automotive research communities.

\section{Acknowledgements}

The authors would like to acknowledge all members of the Computationally Optimised Fuel-Efficient Concept Car (COFEC) project and Advanced Computing Lab(Grid Computing $L a b)$ members for their comments and contributions.

\section{References}

[1] Proceedings Computationally Optimised Fuel-Efficient Concept Car (COFEC) Technofund Project TF0608C073 Volume I, Centre for Automotive Research, Faculty of Engineering and Built Environment, Universiti Kebangsaan Malaysia, 2011.

[2] gLite: EGEE's Next Generation Grid Middleware, http://egee1.eu-egee.org/

[3] CD Adapco Products- STAR CCM http://www.cdadapco.com/products/star_ccm_plus/index.html

[4] About Hyperworks http://www.altairhyperworks.com/HWTemp1.aspx?top nav name= HyperWorks Overview\&item_name=About

[5] OpenFoam http://openfoam.com/

[6] modeFrontier http://modefrontier.com/homeMF.html

[7] LS DYNA http://www.1s-dyna.com/1_pages/11sdyna.htm

[8] Lightweight Middleware for Grid Computing(gLite) http://glite.web.cern.ch

[9] GLITE 3.2 USER GUIDE MANUALS SERIES https://edms.cern.ch/file/722398/1.4/gLite-3UserGuide.pdf

[10] Enabling Grids for E-sciencE (EGEE) http://www.eu-egee.org/

[11] KnowledgeGRID Malaysia PGRADE Portal http://ui01.knowledgegrid.my:8080/gridsphere

[12] KnowledgeGRID ${ }^{\mathrm{TM}}$ Malaysia https://www.knowledgegrid.net.my

[13] Torsten Antoni, Flavia Donno, Helmut Dres, Günter Grein, Gilles Mathieu, Alistair Mills,David Spence, Philippa Strange, Min, Tsai, Marco Verlato Global Grid User Support: The Model and Experience in LHC Computing Grid, http://indico.cern.ch/contributionDisplay.py?contribId=320\&sessionId=7\&confId=048, 2006

[14] I. Stokes-Rees, A. Soroko, C. Cioffi, University of Oxford, UK, A. Tsaregorodtsev, V. Garonne, CPPM, France, R. Graciani, University of Barcelona, Spain, M. Sanchez, University of Santiago de Compostela, Spain M. Frank, J. Closier, CERN, G. Kuznetsov, RAL, UK, Developing LHCb Grid Software: Experiences and Advances, http://www.gridpp.ac.uk/papers/ah04_dirac.pdf, 2004 